\title{
WHO Listed Small Molecule Kinase Inhibitors 2001-2017
}

\author{
Gottfried Sedelmeier ${ }^{\star a}$, Joerg Sedelmeier ${ }^{\star a}$, and Helen Lightfoot ${ }^{b}$
}

The World Health Organization (WHO) publishes quarterly reports listing Internationally recognized Nonproprietary Names (INNs) for substances that are to be marketed as a pharmaceutical. Each INN is also globally recognized as the unique generic name of a drug. The INN contains a specific suffix that defines what compound class the drug belongs to. This poster depicts the chemical structures, biological targets, most advanced indications and associated pharmaceutical companies of small molecule drug compounds with the 'nib' suffix which refers to kinase inhibitors. Kinases comprise one of the largest families of proteins encoded by the human genome. Furthermore, they are one of the most sought-after targets in current pharmaceutical research. Kinases catalyze the transfer of the $\gamma$-phosphate group of ATP onto a protein substrate. This phosphate transfer mediates signal transductions which in turn regulate an array of cellular processes, including proliferation, survival, apoptosis and metabolism. Consequently, cancer is a common indication for which kinase inhibitors are developed. Thirty-seven kinase inhibitors have received FDA approval for the treatment of malignancies, with twenty-six approvals occurring in the last six years. ${ }^{[1]}$

We hope this poster inspires researchers and serves as a springboard of ideas for scientists interested in the development of new kinase-targeting entities.

The poster is available for download: https://chimia.ch/kinase-inhibitors-2017

[1] K. S. Bhullar, N. Orrego Lagarón, E. M McGowan, I. Parmar, A. Jha, B. P. Hubbard, H. P. V. Rupasinghe, Molecular Cancer 2018, 17, 48.

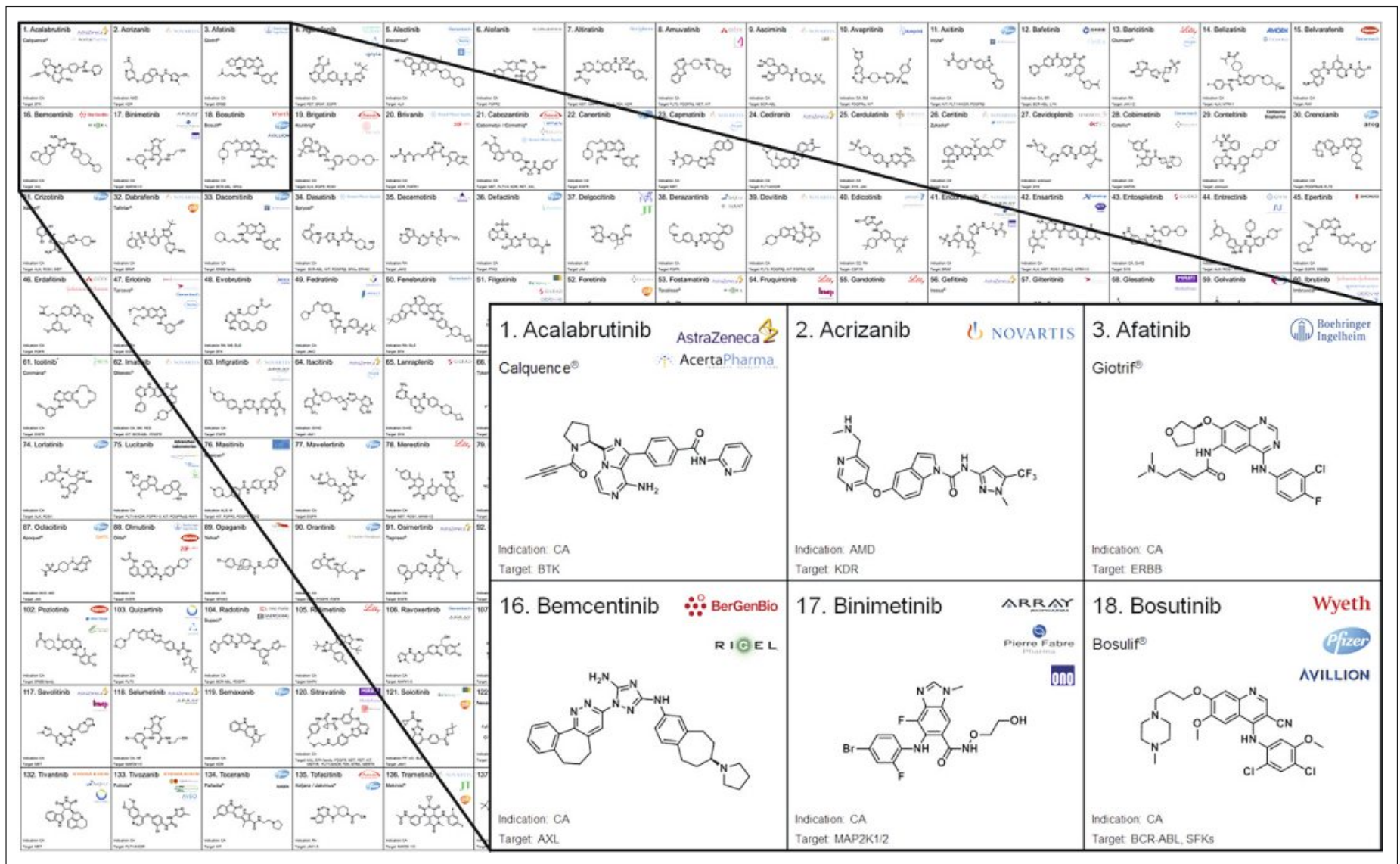

${ }^{\star}$ Correspondence: Dr. G. Sedelmeiera,

Dr. J. Sedelmeier ${ }^{\mathrm{a}}$

E-mail: gottfired.sedelmeier@novartis.com

joerg.sedelmeier@novartis.com

aGDD/TRD/Chemical \& Analytical Development

Novartis Pharma AG, Basel, Switzerland

bHit Discovery, Discovery Sciences, IMED Biotech Unit

AstraZeneca, Alderley Park, UK 